\title{
La copia y la reconstrucción: un recurso visual en las exposiciones de historia del arte moderno desde los años 60 del siglo $X X$
}

\section{On Copies and Reconstructions: A Visual Asset for the Exhibitions of Modern Art History since the 1960s}

\author{
IsABel TeJeda Martín \\ Facultad de Bellas Artes. Universidad de Murcia \\ istejeda@um.es
}

Recibido: 29 de diciembre de 2011

Aprobado: 1 de febrero de 2012

\begin{abstract}
Resumen
Este artículo analiza el hecho de que muchos de los trabajos pertenecientes a las vanguardias históricas de la primera mitad del siglo XX, así como de parte de las neovanguardias han sido paulatinamente reconstruidos desde los años 60 para cubrir las lagunas materiales de algunas exposiciones temporales y de muchos museos. Nos referimos a aquellas obras que nacieron con vocación temporal y efímera, otras que tenían un discurso crítico hacia el atesoramiento de los museos y que desaparecieron años después de ser creadas, así como gran parte de las piezas de carácter ambiental, interactivo y conceptual producidas durante los años 60 y 70 .

Estas copias no suelen destruirse sino que acaban, casi sin excepciones, en las cámaras de colecciones y museos para servir en un previsible futuro tanto para apuntalar los discursos museográficos como para ser prestadas en proyectos temporales ajenos. La paradoja reside en el hecho de que los museos suelen utilizar para las copias los mismos protocolos de conservación que para las obras originales, eliminando con ello parte de la función didáctica que tenían en su origen. Encerrado en vitrinas, este material se protege de las manos, y por tanto de la experiencia, de los espectadores.
\end{abstract}

Palabras Clave: Museografía, Historia, Arte moderno, Educación, Copia.

Tejeda Martín, I. (2012): La copia y la reconstrucción: un recurso visual en las exposiciones de historia del arte moderno desde los años 60 del siglo XX. Arte, Individuo y Sociedad, 24(2) 211-226

\section{Abstract}

This paper analyses the fact that most of the works pertaining to the so-called "Historical Avantgarde"- as well as to some of the Neo - Avant-garde - from the first half of 20th century have been gradually rebuilt since the 1960 s to fill the gaps in some temporary exhibitions, and in many museums. We mean those works that were created with an ephemeral or plain temporary intention; we refer to others that articulated a critical discourse against the compulsive hoarding of museums, as well as to the greater part of works with an environment-like, interactive or conceptual nature which were produced during the 1960 s and the 1970 s.

These copies are not usually destroyed; they end up, almost without exception, in the vaults of museums and collections. And, in the foreseeable future, they are to be employed not only to support a museographical discourse, but also to be loaned for temporary projects of third parties. The paradox lies within the fact that museums tend to use the same set of formalities for preservation that they apply to original works, cancelling then most of the educational values that those copies had in origin. Locked up inside every manner of display cabinet, these materials are protected from the hand and, therefore, sadly absent from the experience of viewers.

Key Words: Museography, History, Modern Art, Pedagogy, Copy.

Tejeda Martín, I. (2012): On Copies and Reconstructions: A Visual Asset for the Exhibitions of Modern Art History since the 1960s. Arte, Individuo y Sociedad, 24(2) 211-226 
Sumario: 1. Introducción, 2. Las vanguardias históricas, 3. El caso del Gabinete de los Abstractos y su re-contextualización junto al Merzbau, 4. Suma y Sigue. La Biennale di Venezia de 1976, 5. Cuando la reconstrucción no es copia. Plight de Josef Beuys en el Centre Georges Pompidou de París, 6. Conclusiones. Referencias.

\section{Introducción}

A partir de los años 60 del siglo XX, el contexto general en que se situaban los artistas contemporáneos de las llamadas neo-vanguardias enfatizaba el concepto y la experiencia por encima del objeto, las obras huían del peso y de la presencia rotunda, y se reivindicaba la inmaterialidad como estrategia para evitar que la radicalidad de sus discursos se convirtiera en un valor de cambio (Foster: 2001, p. 8). Era un momento en el que se hacía preciso legitimar estas experiencias con la construcción de genealogías que las proyectaran hacia el pasado, hacia las vanguardias históricas ${ }^{1}$. Las exposiciones no sólo fueron el dispositivo de visibilidad y producción de estos nuevos lenguajes, sino que también fueron la fórmula elegida para erigir estas genealogías a partir de muestras de carácter histórico. Sin embargo, esta nueva narración de la historia del arte moderno contaba con numerosos vacíos ya que muchas de estas influyentes obras habían sido de carácter efímero o fueron destruidas; el modernismo museográfico que había imperado desde los años 40 en las instituciones de la órbita occidental al amparo del MoMA de Alfred Barr, priorizaba la presencia física del objeto dentro de su relato; de esta manera, había opacado o, al menos, retirado a un puesto subsidiario a aquellas disciplinas y tendencias de las vanguardias de carácter más procesual, ambiental o performativo. Las exposiciones mismas de las vanguardias se contaban entre estas obras temporales.

Durante este proceso de legitimación que se perpetúa hasta el día de hoy, se partió en un principio de documentos gráficos y fílmicos, decidiéndose en ocasiones la reconstrucción de algunas de las obras desaparecidas, así como la copia de otras existentes pero de difícil acceso ${ }^{2}$. Esto, además de haber provocado grandes controversias, subraya en la historización del arte moderno la categoría de la idea por encima del soporte material de la obra.

\section{Las vanguardias históricas}

Si bien se conserva una parte sustancial de las instalaciones y objetos espaciales realizados a partir de los años 70, es importante subrayar que sus antecedentes y muchas de las obras ambientales, performativas u objetuales de los movimientos de las vanguardias históricas desaparecieron casi por completo antes de la Segunda Guerra Mundial. Así sucedió con Porte-bouteilles (1914), Porte-chapeaux (1917) o Fountain (1917) de Marcel Duchamp, Cadeau (1921), Indestructible Object (1923) y Lampshade (1919) de Man Ray, Relieve de rincón (1915), Relieve de rincón complejo (1915) o Contrarrelieve (1917) de Vladimir Tatlin, o las Dada-Puppen (1916) de Hannah Höch ${ }^{3}$. La reconstrucción de algunas de estas obras desaparecidas y la copia de otras que habían sobrevivido a los embates del tiempo, se realizó en ciertos casos de acuerdo con el artista. Durante la producción de la gran retrospectiva que la Tate londinense celebró sobre Marcel Duchamp en 1966 se realizó una copia de Grand verre debido a que el original, en 


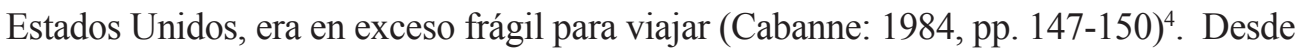
1964 a 1966 se reconstruyeron o copiaron trece objetos que contaron con el beneplácito del artista, entre ellos Fresh Widow, Fountain o el citado Grand verre, hoy en día réplicas mostradas en museos significativos como el MoMA de Nueva York, el Philadelphia Museum of Art, el Centre Georges Pompidou de París, la Tate Modern en Londres, etc 5 .

Sería el recientemente desaparecido artista Richard Hamilton el que realizaría la réplica británica de Grand verre o una nueva versión de Green Box en colaboración con Marcel Duchamp. En realidad, las copias fueron realizadas por Hamilton y Duchamp, encantado por el resultado, decidió firmarla, pero le pidió al británico que estampara su nombre junto al suyo ${ }^{6}$. Muchas décadas después, cuando el tiempo comienza su proceso de legitimación histórica, llegaría el turno del propio Richard Hamilton que dio su visto bueno para que se reconstruyera Fun House, obra instalativa que marcó el inicio del pop art británico y realizada en el año 1956 junto a John Voelcker y John McHale para el proyecto expositivo This is Tomorrow (Whitechapel Art Gallery) (Tejeda: 2006 b) ${ }^{7}$. Las reconstrucciones de obras de las vanguardias históricas han sido constantes desde entonces. En algunos casos se han entendido como una fórmula pertinente para recuperar obras perdidas que pudieran funcionar como un dispositivo didáctico que cubriera ciertas lagunas temporales en los museos; en otros, los historiadores o artistas que las han llevado a cabo las han entendido como un vehículo para la investigación y análisis del pensamiento de sus autores (Leleu: 2005, p. 137); en los menos, para ahorrarse los carísimos y peligrosos transportes trasatlánticos o para salvar los imponderables de un préstamo rechazado. Vamos a citar aquí algunas de las copias y reproducciones que afectan a instalaciones o trabajos ambientales.

\section{El caso del Gabinete de los Abstractos y su re-contextualización junto al Merzbau}

Las grandes exposiciones internacionales de cuadros semejan un zoo donde el rugido de miles de fieras diferentes asalta al visitante al mismo tiempo. En mi sala de exposiciones los objetos no deberían atacar simultáneamente al espectador. Si antaño se favorecía cierta pasividad del espectador al pasar antes las paredes llenas de cuadros, el presente proyecto pretende estimular su actividad. Este debería ser el objeto de nuestra sala. (El Lissitzky: 1929a, p. 132).

A partir de esta reflexión, el artista constructivista ruso El Lissitzky diseñó una "máquina de visión" como espacio expositivo para presentar el arte entonces más contemporáneo en el Landesmuseum de Hanover (Nobis: 1998, p. 145). Una "máquina" para la que la intervención del espectador era imprescindible y que estaba dotada de específicos estudios lumínicos, diferentes texturas para cada muro, paneles corredizos y estructuras geométricas que parcelaban el espacio expositivo (El Lissitzky: 1929 b, p. 118). Este espacio estaba situado al final de la segunda planta del museo que había diseñado su director, Alexander Dorner, ocupando en total 20 metros cuadrados. Se erigía como conclusión de la historia del arte que reunía el museo a partir de una idea de progreso concretada en su recorrido cronológico, de esta manera el presente quedaba integrado en la narración. Así, el súmmum artístico del Landesmuseum se encontraba en las obras de Picasso, Mondrian, Leger, Archipenko, 
Pevsner, Schwitters, Picabia, Moholy-Nagy, Vordemberge-Gildewart, Schlemmer, Baumeister, Kandinsky, Jawlensky, Klee, Feininger, Delaunay, Gleizes, Marcousis, Gabo, etc.

El Gabinete de los Abstractos (Kabinett der Abstrakten) fue desmontado en 1937 por el gobierno nacional-socialista. El Lissitzky estaba por entonces en Moscú -moriría en 1941 de tuberculosis- dedicándose de lleno al diseño de los pabellones soviéticos que servían de propaganda estalinista en las manifestaciones industriales internacionales (Leering: 1990, p. 60). Alexander Dorner asistió apenado a la destrucción del trabajo que había ocupado su vida durante casi dos décadas: su intento de construir un museo moderno. Acabaría emigrando a los Estados Unidos (Nobis: 1998, p. 154). Algunas de las piezas de los fondos de arte contemporáneo del Landesmuseum de Hanover fueron vendidas en el extranjero y otras fueron destruidas por los nazis, dándose por perdida la mayor colección de este tipo que había por entonces en Alemania. Según Dietrich Helms, en la campaña contra el "arte degenerado" desaparecieron más de 250 obras de arte (Helms: 1963, p. 144).

Pese a su desaparición, el Gabinete de los Abstractos seguía siendo una pieza fundamental para entender la entrada de la modernidad en Alemania. Por ello, en la primera exposición que supuso un estudio del arte moderno en la Hanover de la República de Weimar, la muestra Los años 20 en Hanover, se decidió producir un gran mural fotográfico que, casi a tamaño natural y formando una ' $L$ ', permitía que el visitante se hiciera una idea de cómo había sido aquella sala final del museo. La exposición tuvo lugar en 1963 y las piezas que entonces se pudieron recuperar para la muestra y que habían formado parte de la sala diseñada por El Lissitzky, se colgaron, sin embargo, manteniendo los estándares expográficos del cubo blanco rodeando la reproducción (Helms: 1963).

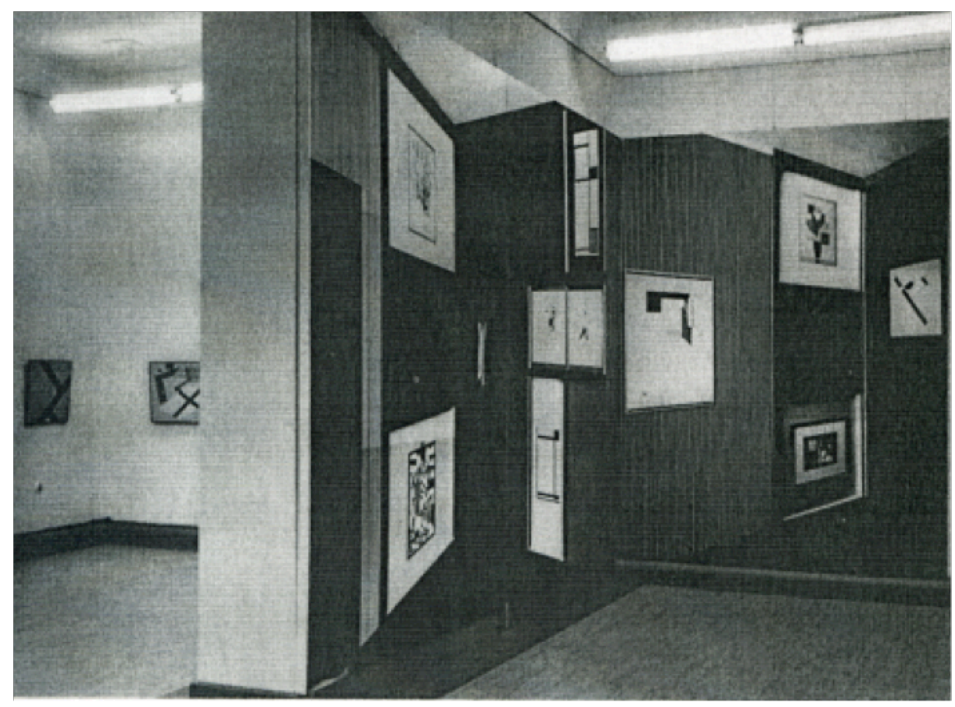

Figura 1. Panel fotográfico reproduciendo el Gabinete de los abstractos para la exposición Los años 20 en Hanover, que tuvo lugar en esta ciudad en 1963. Fuente de la imagen (Helms: 1963, p. 143). 
El siguiente paso tuvo lugar en 1968. El gabinete se reconstruyó en la sala 45 del viejo museo, centro que había pasado a denominarse Museo Regional de la Baja Sajonia. La reconstrucción tuvo en cuenta, amén del reportaje fotográfico que se había realizado en 1927, los testimonios de aquellos que habían visitado el gabinete, en especial las indicaciones de Lydia Dorner, viuda del antiguo director del Landesmuseum (Forgács: 2003).

Finalmente la obra se integró en el nuevo edificio del Museo Sprengel de la misma ciudad, encontrándose en una nueva copia de 1979, desde principios de los años 80, junto a la reconstrucción del Merzbau, una intervención de escultura, arquitectura y pintura que Kurt Schwitters había montado en los distintos hogares que habitó: su casa en Alemania desde 1927 a 1937 (destruida por los bombardeos aliados durante la Segunda Guerra Mundial), su hogar noruego (devorado por un incendio en 1951) y finalmente el inglés (de este último es del único que se conservan restos originales que Richard Hamilton trasladó en 1965 de su emplazamiento original en un viejo granero a la Hatton Gallery en Newcastle-upon-Tyne).

La reconstrucción actual de este espacio "bricolado" situada en la planta baja del museo data de los años 1981-83 y fue realizada por Peter Bissegger a instancias de Harald Szeemann, que la precisaba para su exposición Der Hang zum Gesamtkunstwerk. Bissegger se ha especializado desde entonces en la producción de réplicas realizando la reconstrucción de otras tres esculturas de Schwitters, incluida Die grosse MERZsäule, así como de los figurines de ballet de Oskar Schlemmer ${ }^{8}$. En el caso del Merzbau, creemos que la reconstrucción del Museo Sprengel ofrece menos garantías de veracidad que El gabinete de los abstractos, ya que Kurt Schwitters nunca entendió su casa de ocho habitaciones como un espacio acabado, sino en continua mutación; esto significa que esta versión muestra congelado un instante y un único punto de vista proveniente de una sola toma fotográfica, una imagen capturada por Wilhelm Redemann en 1933. La experiencia del espectador en esta reconstrucción es comparable a la contemplación que propicia el atelier Brancusi, también replicado, que se levanta hoy a los pies del Centre Georges Pompidou en París. El hecho de que la obra de El Lissitzky y la de Schwitters se muestren hoy pared con pared no deja de ser un milagro de la re-contextualización museística, amén de que convierte en portátiles las obras ambientales: otra versión del Merzbau, esta vez de viaje, fue reconstruida en 1988, de nuevo por Bissegger, para participar en exposiciones de carácter itinerante, habiéndose presentado hasta hoy en un total de veintitrés muestras. Peter Bissegger, además, tiene el copyright de estas réplicas, lo que, en cierto modo, pone también en cuestión el concepto de autoría única o de originalidad. 
।

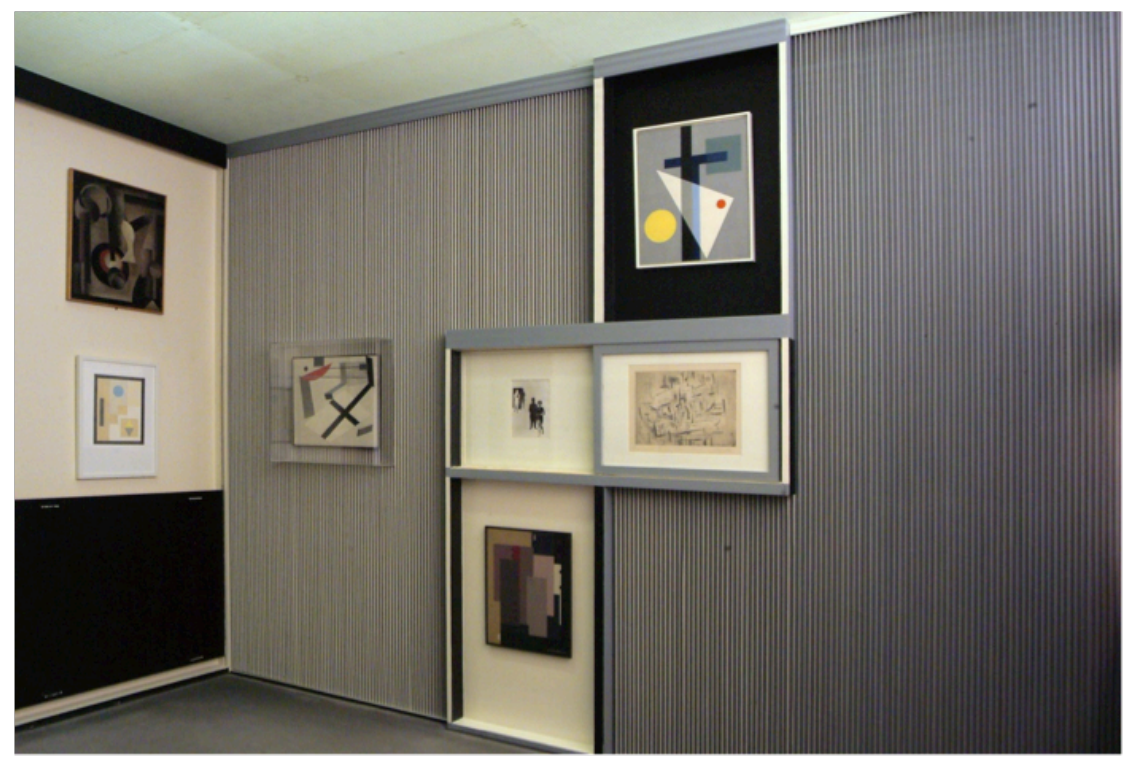

Figura 2. El Lissitzky. Kabinett der Abstrakten.1928 (reconstruido en 1979 por el Sprengel Museum Hannover). (C) VEGAP, Madrid. Fotógrafo: Michael Herling / Aline Gwose, Sprengel Museum Hannover. Imagen cedida por el Sprengel Museum Hannover.

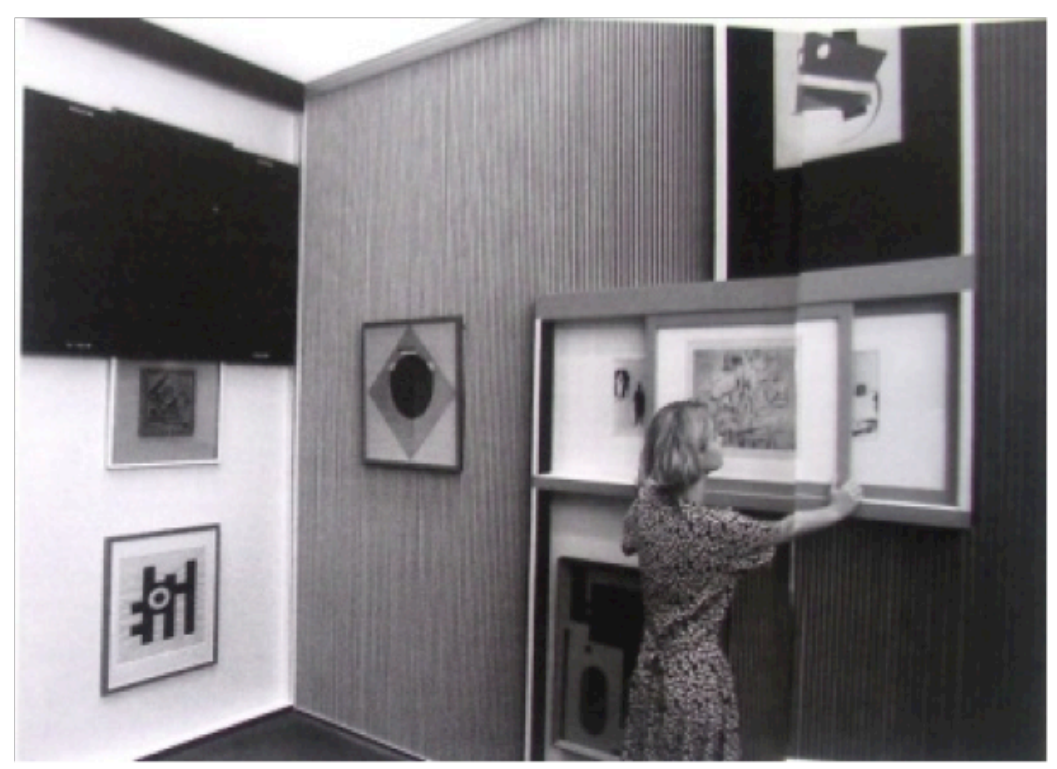

Figura 3. Reconstrucción del Gabinete de los abstractos de 1979 con una usuaria. Fuente de la imagen (Nobis: 1998, p. 153). 


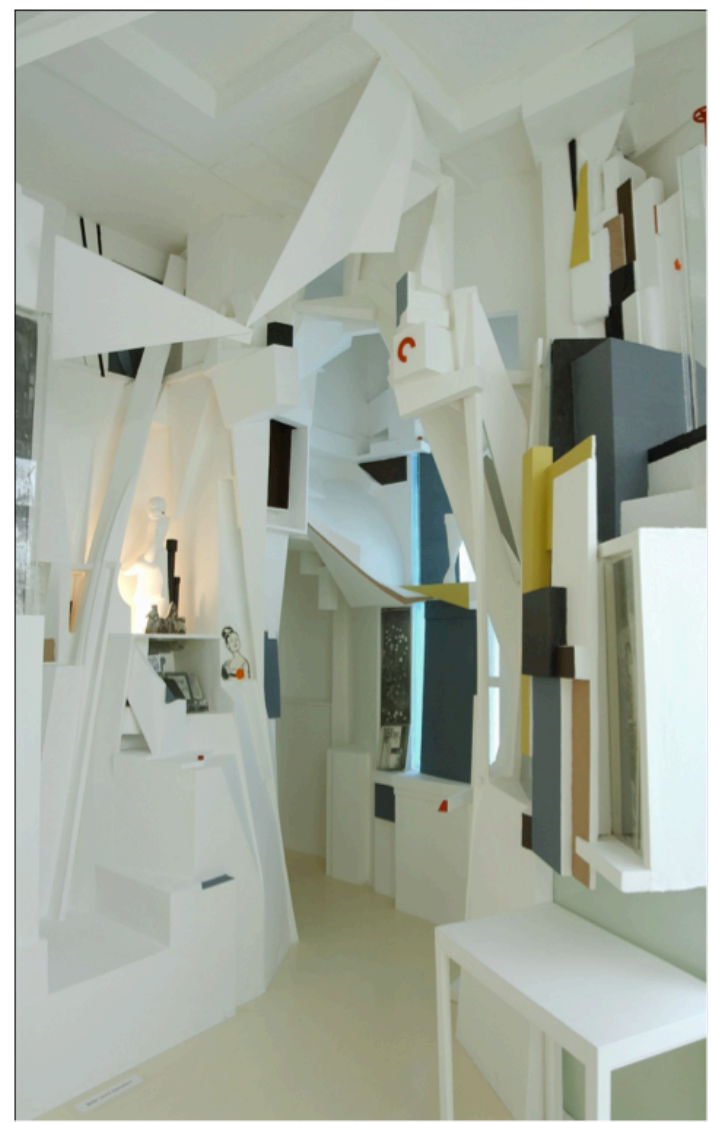

Figura 4. Reconstrucción del Merzbau de Kurt Schwitters de 1933 por Peter Bissegger. 1981-1983. Sprengel Museum Hannover. (C) Peter Bissegger. Fotógrafo: Michael Herling / Aline Gwose, Imagen cedida por el Kurt Schwitters Archiv im / en el Sprengel Museum Hannover.

\section{Suma y Sigue. La Biennale di Venezia de 1976}

El clímax de las reconstrucciones y de las reproducciones llegaría con la canonización de las instalaciones en la Biennale di Venezia de 1976 (Tejeda: 2006a, pp. 47-49). Germano Celant, comisarió una muestra de exposiciones e instalaciones en el pabellón central de I Giardini bajo el título Ambiente/Arte. La acepción de ambiente, previa a la hoy más difundida de instalación, establecía según Celant "una serie de relaciones físicas y perceptivas entre el espacio y la investigación artística[...] concibiendo el espacio no como vaso o recipiente que acoge pasiva o indiferentemente una estructura, sino como un agente más de su intervención" (Celant: 1976, p. 189)9

El objetivo del crítico y comisario italiano era legitimar la obra de sus contemporáneos, como Daniel Buren, Dan Graham, Bruce Nauman, Sol LeWitt, Kounellis, Vito Acconci o Joseph Beuys, por lo que les proporcionó una base 
genealógica que iniciaba históricamente con los futuristas italianos, los constructivistas rusos, los neoplasticistas, los artistas dadá o los surrealistas. En este sentido no sólo recogía obras en el sentido tradicional del término, sino que también le interesaba subrayar cómo habían sido expuestas. Como si se tratara de una continuidad lógica, la exposición seguía su relato a partir de las obras de los artistas de la generación anterior como Jackson Pollock, Andy Warhol, Lucio Fontana, Allan Kaprow o Yves Klein, entre otros, para finalizar con las instalaciones de los creadores más jóvenes que estaban encabezadas por Blinky Palermo en la sala 8 y terminaban con Michael Asher en la número 20. El peso del pabellón quedaba así compensado: la mitad para el pasado histórico y la otra mitad para las producciones nuevas realizadas especialmente para la Biennale.

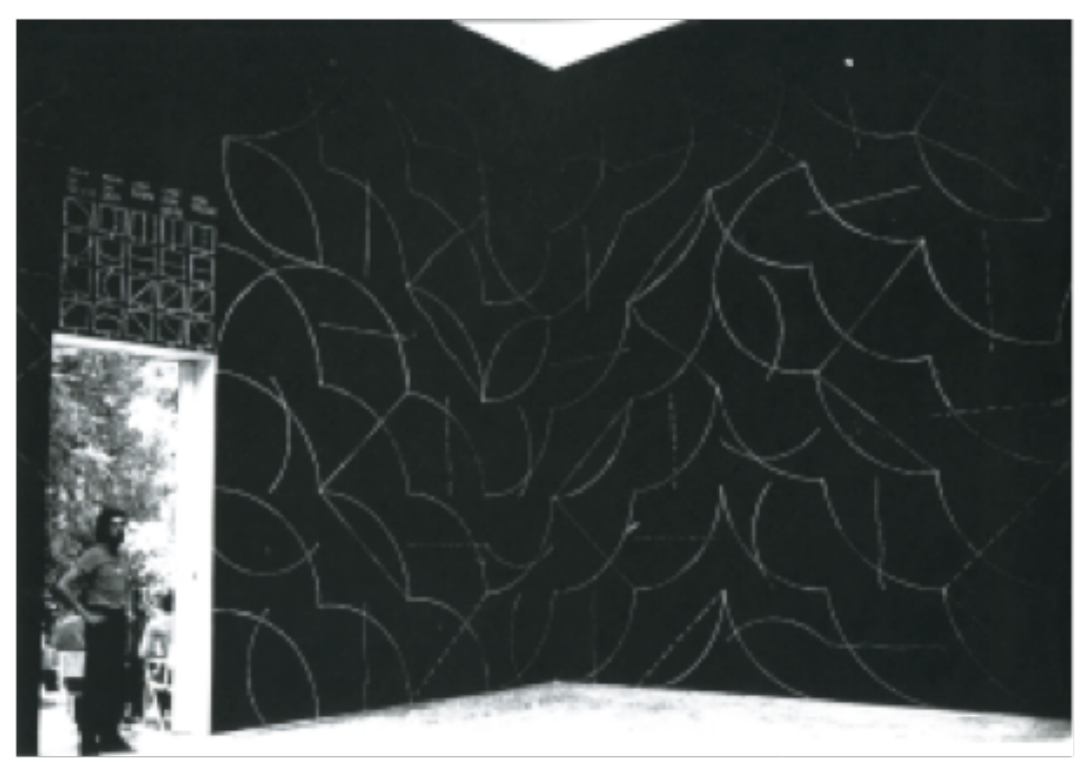

Figura 5. Intervención de Sol LeWitt en la Biennale di Venezia de 1976. Fotografía en The Hyman Kreitman Research Centre for the Tate Library and Archive

Pero Germano Celant se encontró con el problema de que casi no tenía obras ya que

[...] la totalidad de los trabajos ambientales [denominaba ambientes a las protoinstalaciones y obras espacialistas de las primeras vanguardias] fue destruida de tal manera que su conocimiento depende de la reconstrucción y de los documentos fotográficos (Celant: 1976, p. 190) ${ }^{10}$.

Las razones por la que muchas de estas piezas hubieran desaparecido son muy variadas. Por un lado, muchas habían nacido para participar en el marco de eventos marginales y anti-institucionales por lo que perdurar no estaba dentro de su ideario. Además, los entonces jóvenes museos de arte contemporáneo que empezaron a 
crearse a partir de finales de la década de los años 20 entendían como colección un conjunto de objetos de fisicidad incuestionable y realizados con materiales más o menos perdurables, fundamentalmente pinturas y esculturas ${ }^{11}$. El propio mercado no empezó a interesarse por este otro tipo de manifestaciones producidas durante las vanguardias hasta la década de los años 60 , y ya entonces era tarde, casi todo había desaparecido por desinterés, desidia o por la propia guerra.

[...] el procedimiento ambiental corroe las estructuras en las que predomina el intercambio, esta práctica es evitada tanto por los medios informativos como por los distintos operadores, lo que tiene como resultado su marginalidad (Celant: 1976, $p$. 190).

Por ello, Celant reconstruyó y reprodujo algunas de las más significativas piezas de las vanguardias históricas que habían desaparecido mezclando en la exposición piezas originales de los artistas más jóvenes junto a dispositivos de dos tipos: en primer lugar podemos citar las reproducciones que resultaban de la ampliación de fotografías originales de los montajes y que se presentaban en forma mural; en segundo lugar, las reconstrucciones propiamente dichas.

En el primer caso se encontraban las fotografías murales que, siguiendo la estela de la exposición de Hanover en 1963, ampliaban sin llegar al tamaño natural de aquella muestra, los documentos gráficos del Gabinete de los abstractos, del pasillo de los maniquíes de las Plus belles rues de Paris de la muestra internacional surrealista parisina de 1938 o del intrincado enredo de hilos duchampiano de First Papers of Surrealism (Nueva York, 1942).

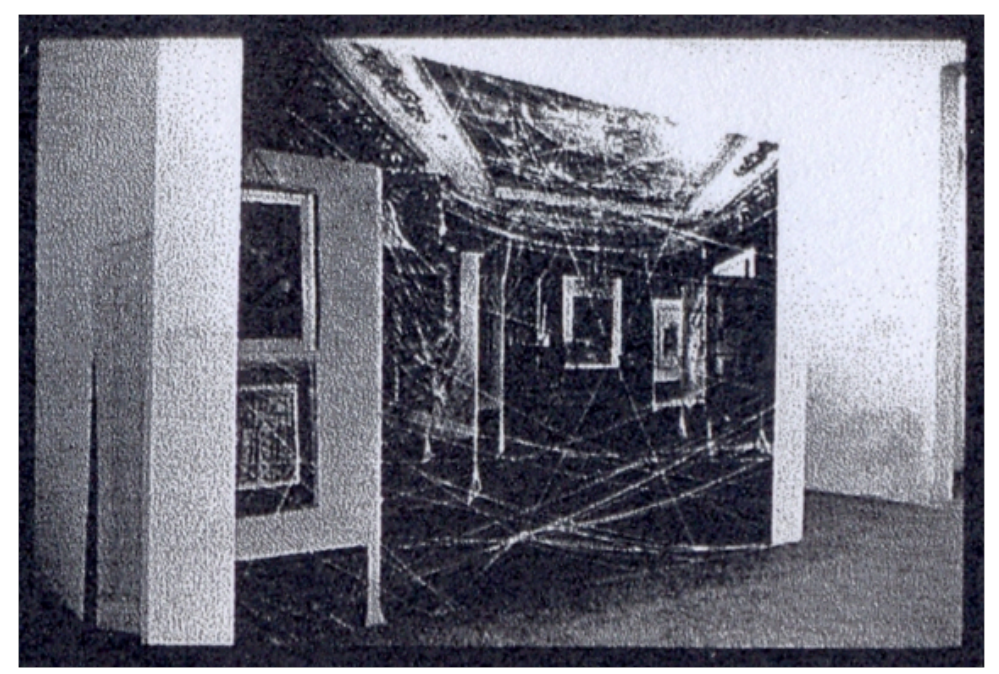

Figura 6. Panel fotográfico que reproducía dos imágenes de First Papers of Surrealism (1942) en la Biennale di Venezia de 1976. Fotografía perteneciente a The Hyman Kreitman Research Centre for the Tate Library and Archive. 
Germano Celant también decidió realizar un arriesgado montaje a partir de una obra original del expresionista abstracto americano Jackson Pollock y cuatro fotografías murales. De forma heterodoxa colocó la tela, en lugar de colgada en la pared, acostada sobre el suelo, mientras forraba los muros adyacentes con ampliaciones de las famosas fotografías que Hans Namuth había tirado en el estudio del pintor en 1950 mientras éste pintaba. El historiador italiano estaba, en este caso, ya no sólo reconstruyendo, sino interpretando. Remitía, de esta manera, la pintura de Pollock no a su contemplación final sino a la procesualidad de su momento de realización y a la ocupación física, espacial, que el pintor americano hacía de la tela para producirla. También Celant reivindicaba una horizontalidad en la presentación de la obra del pintor que fue posteriormente analizada por Rosalind Krauss en $E l$ Inconsciente óptico (según teorizaba la ensayista norteamericana, la "horizontalidad del acontecimiento" tenía una "proyección especular de la organización del cuerpo del observador" que la sublimación de la lectura óptica modernista cancelaba para transformarla en un "plennum visual") (Krauss: 1997, pp. 289-325).

A partir de estas primeras reconstrucciones italianas y alemanas se abrió la veda, sobre todo cuando comenzaron a proliferar los nuevos museos tras la eclosión de los denominados centros de arte en los años 80 . En la actualidad, suele ser bastante común encontrarse tanto en exposiciones temporales como en los circuitos permanentes de los museos las copias realizadas desde los años 60 .

Sin embargo, y para no llegar al límite de la reconstrucción, ya que se considera, como en fotografía, que el objeto original, el vintage, siempre es más valioso que su reelaboración posterior aun cuando ésta se lleve a cabo con el consentimiento del artista, los museos han desarrollado sistemáticamente estrategias de conservación que parten del análisis de materiales que hasta hace cuarenta años eran extra-artísticos. En ocasiones se ha llegado a la paradoja de congelar el proceso de desaparición de una pieza y, por tanto, de destruir su sentido como obra procesual, situación obsesiva que persigue, por ejemplo, a las obras de la artista germano americana Eva Hesse (Tejeda: 2006a, pp. 42-43). Lo curioso y llamativo es que estos extenuantes protocolos de conservación preventiva se han hecho extensibles a las reconstrucciones y copias que se muestran protegidas dentro de vitrinas o tras catenarias. Para saber que no se trata de la obra original sólo suele ofrecerse al visitante, de forma algo críptica, un dato en la cartela: se incluye, después de la fecha de su creación y tras un guión, la fecha en que fue reconstruida. La inquietud, pues, por conservar y custodiar los objetos no siempre brinda resultados acertados.

En este sentido, la larga lista de reproducciones y reconstrucciones que podemos citar pone en evidencia hasta qué punto es una práctica corriente y ha sido legitimada por el sistema museístico. Además de las obras citadas, el Espacio Proun de El Lissitzky (1923) y Habitación con flores de Theo van Doesburg (1925) fueron reconstruidos en 1965 y 1968 para el Van Abbemuseum de Eindhoven; el Salon de Madame B. de Piet Mondrian fue construido en 1970 a partir de un dibujo de 1926; el Licht-Raum-Modulator (1930) de László Moholy-Nagy fue montado de nuevo en 1970 para el Stedelijk van Abbemuseum de Eindhoven; el Modelo de Monumento a la III Internacional (1919) de Vladimir Tatlin, fue reconstruido por el Centre Georges Pompidou en 1979 a partir de fotografias; Isabel Kork y Barbara Kugel volvieron a 
coser las desaparecidas Dada-Puppen (1916) de Hannah Höch en 1988 siendo en la actualidad atesoradas por la Berlinische Galerie - Landesmuseum für Moderne Kunst, Photographie und Architektur ${ }^{12}$; el Bar Aubette (1926-28) de Sophie TaeuberArp fue reconstruido en 1998; y la Kunstsammlung de Weimar hizo lo propio en 2009 con Turm des Feuers (1920) de Johannes Itten (Troy: 1983, pp. 60 y 124-128; Leleu: 2005, p. 85; VV.AA: 2004, p. 110).

\section{Cuando la reconstrucción no es copia. Plight de Josef Beuys en el Centre Georges Pompidou de París}

Un caso bastante llamativo de las neovanguardias en el sentido de la aplicación de dispositivos de conservación no adecuados es el de la obra Plight de Josef Beuys; esta pieza se muestra dentro del circuito de la colección permanente del Centre Georges Pompidou de París. Plight está conformada por dos habitaciones forradas por doscientas ochenta y cuatro balas de fieltro de un metro y medio de alto, un termómetro, una pizarra, un piano de cola y un único acceso bastante bajo que obliga al espectador a agacharse para poder entrar a la instalación. Se trata de una de las raras obras de Beuys que mantienen la vitalidad de la experiencia, centrada, en este caso, en producir una sensación de aislamiento (Bernárdez: 1999, p. 71): el fieltro provoca una impresión térmica distinta, un olor característico, así como una acústica especial debido a que es un aislante sonoro, lo que generalmente incita al silencio del visitante. De esta manera lo definía el propio Beuys cuando en 1986 dirigió su instalación en el museo, tras haber presentado una primera versión un año antes en la galería londinense Anthony d'Offay ${ }^{13}$ :

Otro significado de la pieza, en este caso positivo, es proteger al ser humano del peligro. De esta manera aparece la idea de la sala de conciertos, sin resonancia, es decir, totalmente negativa, concebida como la demostración de la existencia de una frontera donde todo se articula alrededor de un punto crítico. Si este punto se mueve, todo cambia, incluso el significado del arte, que tiende, como sabemos, al cambio radical y completo del ser humano comenzando por la conciencia que tiene de sí mismo. (VVAA: 1996) $)^{14}$.

Hasta hace unos años, el visitante podía entrar libremente y caminar por este espacio, sin embargo, en la actualidad el museo ha decidido colocar nada más entrar en la instalación una catenaria de metal y cristal a modo de pequeño balcón. La razón no podía encontrarse en la adopción de un protocolo de conservación material original ya que Beuys se sirvió del fieltro prácticamente en su acabado industrial y sin más manipulación, lo que permitiría, teóricamente, su renovación de tanto en tanto. La lógica que ha impulsado al museo a tomar esta radical terapia es de origen pecuniario: los visitantes estropeaban los cilindros de fieltro por lo que el museo se veía en la necesidad de cambiarlos de tanto en tanto, suponiendo un gasto extra para su presupuesto. Con esta catenaria, se condena a Plight a ser contemplada y no a ser experimentada, robando al espectador esa sensación de aislamiento, de experiencia sensitiva y no sólo óptica, que intentaba propiciar Beuys. La aplicación de un dispositivo de visión típicamente modernistas la convierten, por tanto, en una pieza modernista. 


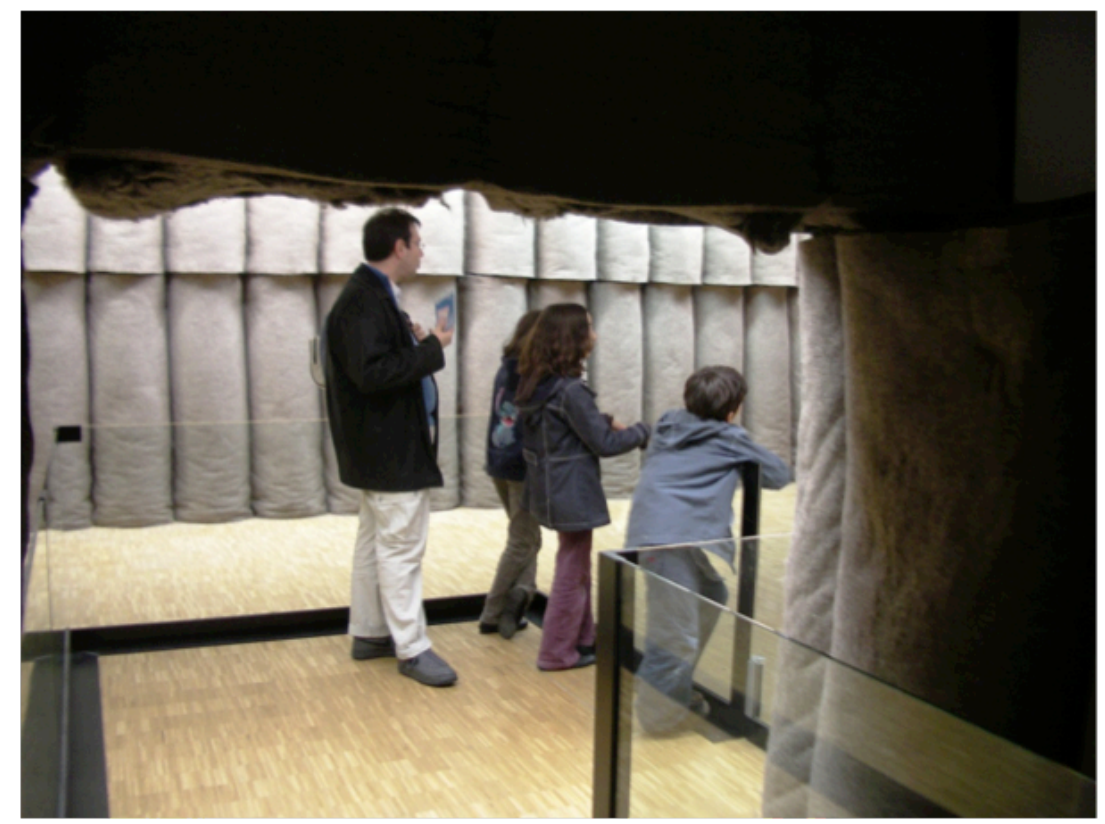

Figura 7. Montaje actual de Plight de Josef Beuys en el Centre Georges Pompidou, París. Fotografía de Isabel Tejeda tomada en la presentación de 2008.

\section{Conclusiones}

Las reconstrucciones han venido planteando la fascinante problemática de la unicidad y originalidad de la obra frente a su posible repetición. Se considera que en las obras espaciales el aura de lo original, ese aquí y ahora sobre el que reflexionara Walter Benjamin (Benjamin: 1989), tiene escasa importancia frente a las posibilidades que abre para conocimiento del arte contemporáneo su rescate genealógico como dispositivos para la condensación de la experiencia.

Resulta sintomático que algunas de estas reconstrucciones estén en las salas permanentes de los museos y que, fundamentalmente, los protocolos de conservación desplegados a su alrededor sean idénticos a los que el museo implementa para las obras originales. También lo es que, en piezas en cuyo programa se encuentra la reconstrucción al no partir del "toque" que da la mano del autor, sino que participan de la importancia del concepto -sirviéndose para ello de las formas básicas del material en su producción industrial- también se asuman dichos protocolos.

Algunas de las obras analizadas fueron creadas como Gesamtkunstwerke (Obra de arte total) de la primera mitad del siglo XX, ambientes en los que no siempre existían límites marcados entre el espacio del espectador y de la obra de arte; de esta manera se lograba su confluencia en un mismo ámbito: podían tocarse y manipularse; la experiencia era la prioridad. 
Si no pueden ser tocadas y experimentadas, estas reconstrucciones y copias dejan de ser utilizadas como recursos pedagógicos -una función que facilita el conocimiento y la comprensión de estas obras al tiempo, la otra es conservar su forma para la historia-, para convertirse en piezas, de esta forma se neutraliza parte del programa y realidad de algunas vanguardias en su anti-objetualidad, su rechazo al sistema mercantil del arte y su desdén por la conservación museológica. Estas obras pueden sufrir un proceso de invalidación al pasar de un estatus de recurso didáctico a pieza "museificada" durante el proceso de historización y legitimización.

Por otra parte, se debe subrayar que, al igual que no se puede aprender dos veces la misma cosa, resulta imposible imitar la razón de ser por la que históricamente nacieron los trabajos ambientales: la experiencia. La realidad que se enmarca deja de serlo. Las cápsulas del tiempo no existen. El hic y nunc de los que hablara Walter Benjamin sólo se produce una vez. Sin embargo, estos cuantiosos y complicados protocolos de conservación preventiva que los museos despliegan sobre sus réplicas y reconstrucciones acaban poniendo en cuestión sus teorías sobre la obra de arte en la época de su reproductibilidad técnica: las cámaras de los museos, así como el pedestal invisible en el que se convierte cada una de sus salas, dotan a estos nuevos soportes de parte del respeto sacrosanto que tenían sus predecesores originales aun sin haber participado jamás en el aquí y ahora referido por el pensador alemán (Benjamin: 1989). El sistema es perverso: de alguna manera revierte el aura y esto sin que ni tan siquiera sea preciso que actúe el valor histórico o valor de antigüedad que analizara Aloïs Riegl (Riegl: 1987). Muchas de estas copias no llegan a tener ni una veintena de años, quedándoles, pues, tan sólo su "valor instrumental".

Ante la desaparición material el intento de reconstruir la experiencia se sitúa entre la aporía y la nostalgia, no obstante, como dispositivo didáctico suficientemente señalado, y utilizado como un recurso expográfico, que no como obra, puede suplir las lagunas en un discurso, el del museo, que pretende ofrecer una posible lectura de la historia del arte y cuyos dispositivos deben servir para el estímulo del conocimiento (Huerta, De la Calle: 2007). Por ello, creemos que estas reconstrucciones deben entenderse, pese a su falta de autenticidad, como un resto más de la historia que nos es ofrecido para vivir otra experiencia contemporánea muy distinta: el ritual de la visita al museo.

\section{Referencias}

Benjamin, Walter, (1989). La obra de arte en la época de su reproductibilidad técnica, en Discursos interrumpidos I, Madrid, Taurus.

Bernárdez, Carmen, (1999). Josef Beuys, San Sebastián, Nerea.

Cabanne, Pierre, (1984). Conversaciones con Marcel Duchamp, Barcelona, Anagrama.

Celant, Germano, (1976). Ambiente/Arte, en La Biennale di Venezia. Environement. Participation. Cultural Structures, Venecia, Alfieri Editori d'Arte, vol. I.

Forgács, Éva, (2003). Definitive Space: the Many Utopias of El Lissitzky's Proun Room en Perloff, Nancy; Reed, Brian, Situating El Lissitzky, Vitebsk, Berlín, Moscú, Los Angeles, Getty Publications. 
Foster, Hal, (2001). El retorno de lo real. La vanguardia a finales de siglo, Madrid, Akal Ediciones.

Guasch, Anna Maria, (1997). El arte del siglo XX en sus exposiciones. 1945-1995, Barcelona, Ediciones del Serbal.

Helms, Dietrich, (1963). The 1920's in Hannover, en Art Journal, Vol. 22, n. 3.

Hohlfeldt, Marion, (1993). Plight de Joseph Beuys, en Cahiers du Musée National d'Art Moderne, n. 43.

Huerta, R., De la Calle, Román, (2007). Espacios estimulantes. Museos y educación artística, Valencia, Generalitat Valenciana, UV.

Leering, Jean, (1990). El dilema de Lissitzky. Respecto a la obra posterior a 1927, en El Lissitzky (1890-1941), arquitecto, pintor, fotógrafo, tipógrafo, Madrid, Fundación Caja de Pensiones, Stedelijk Van Abbemuseum de Eindhoven.

Leleu, Nathalie, (2005). Mettre le regard sous le contrôle du toucher. Répliques, copies et reconstitutions au XXe siècle: les tentations de l'historien de l'art en Les Cahiers du Mnam, no 93, pp. 84-103.

Leleu, Nathalie, (2007). Je crois que la peinture meurt, comprenez-vous. Répliques et reconstitutions de / par / pour / Marcel Duchamp, en Restauration et nonrestauration en art contemporain (Breuil, Marie-Hélène dir.), Rouen, Musée des Beaux Arts de Rouen, pp. 127-148.

Lissitzky, El, (1929). Espacios de demostración en Lissitzky, El, La reconstrucción de la arquitectura en la URSS, Barcelona, Gustavo Gili, 1970.

Lissitzky, El, (1929). La configuración plástica de la exposición electrónica Victoria sobre el Sol en Lissitzky, EL, La reconstrucción de la arquitectura en la URSS, Gustavo Gili, Barcelona, 1970.

Krauss, Rosalind E., (1997). El inconsciente óptico, Madrid, Técnos.

Nauman, Francis, (2004). Marcel Duchamp. L'argent sans objet, París, L'Echoppe.

Nobis, Beatrix, 1998: El Lissitzky. L'espace des Abstraits pour le Musée Provincial de Hanovre, 1927.-1928 en VV.AA., L'art de l'exposition. Une documentation sur trente expositions exemplaires du Xxe siècle, París, Éditions du regard.

Riegl, Aloïs, (1987). El culto moderno a los monumentos. Caracteres y origen, Madrid, Visor.

Sourieau, Étienne, (1998). Diccionario Akal de estética, Madrid, Akal.

Tejeda, Isabel, (2006). El montaje expositivo como traducción. Fidelidades, traiciones y hallazgos en el arte contemporáneo desde los años 70, Madrid, Editorial Trama, Fundación Arte y Derecho.

Tejeda, Isabel, (2006). Pasajes de espacio, tiempo y espectador: instalaciones, nuevos medios y piezas híbridas en la colección del IVAM en Instalaciones y nuevos medios en la colección del IVAM. Espacio, tiempo y espectador (Isabel Tejeda ed.), Valencia, IVAM, pp. 27 a 67.

Troy, Nancy J., (1983). The De Stijl Environment, MIT Press.

VV. AA., (1989). Dadá y constructivismo, Madrid, Centro de Arte Reina Sofía.

VV.AA., (1996). MNAM, La Collection I, Acquisitions 1986-1996, éditions du Centre Georges-Pompidou, Paris.

VV. AA., (2004). Hannah Höch, Madrid, Museo Nacional Centro de Arte Reina Sofía, Aldeasa. 


\section{Recursos electrónicos}

http://www.bissegger.com/

http://www.centrepompidou.fr/

http://www.sprengel-museum.de

\section{Notas}

1. Nos servimos en este sentido del término acuñado por Hal Foster (Foster: 2001, p. 8). Para Foster la neo-vanguardia es "el arte desde 1960 que recupera inventos de la vanguardia con fines contemporáneos (por ejemplo [sigue], el análisis constructivista del objeto, la manipulación de la imagen por el foto-montaje, la crítica premeditada de la exposición".

2. Según el Diccionario Akal de estética, la copia o la réplica "se distingue de la reproducción en que exige la intervención de un nuevo realizador, que efectúa una segunda obra a partir de la primera; por ello, la manera en que se haya visto, leído, comprendido, sentido el original, así como la personalidad de sus gestos (ductus de escrito del que escribe, pincelada del pintor, etc.) impedirá que la copia sea nunca rigurosamente idéntica a este original. La reproducción emplea procedimientos mecánicos (físicos y/o químicos: fotografía, moldeado, etc.) para intentar obtener un doble exacto del original. Ciertos procedimientos, tales como el calco, la cámara clara, se sitúan en la frontera de las dos nociones" (Sourieau: 1998, p. 363). La reconstrucción, por su parte, es la construcción que se hace de nuevo de una cosa destruida o deshecha.

3. Lampshade fue reconstruida en 1959. Las piezas de Tatlin las ha ido reconstruyendo Martyn Chalk entre 1979 a 1989, y pertenecen a Annely Juda Fine Arts de Londres (VV. AA.: 1989, pp. 153-156).

4. La exposición contó con la ayuda y beneplácito del artista.

5. Ocho de estos ready-made fueron objeto de una edición que fue realizada por el marchante italiano de Duchamp, Arturo Schwarz. Los ready-made, producían la idea de "contradicción, que es algo que no ha sido nunca demasiado explotado", decía el propio autor francés sobre su trabajo (Nauman: 2004, p. 16). [Traducción de la autora]. En realidad, Marcel Duchamp no hacía más que insistir en la idea por encima de su materialidad como objeto único, pese a que, con ello, volviera a producir de nuevo "cosas"

6. Por entonces, y junto a innovadoras propuestas personales, Hamilton colaboraba diseñando exposiciones. Además de participar en la muestra de Marcel Duchamp, lo hizo en un proyecto de carácter retrospectivo sobre Francis Picabia (1964).

7. Se reconstruyó en 1987 para el Institute for Art and Urban Resources (The Clocktower) en Nueva York en la exposición (The Pop Project). This is Tomorrow Today. The Independent Group and British Pop Art comisariada por Brian Wallis y Thomas Finkelpearl. (Guash: 1997, p. 53).

8. El Merzbau de Schwitters fue reconstruido en la década de los años 80 por el Sprengel Museum de Hannover; lo llevó a cabo Peter Bissegger siguiendo la iniciativa de Harald Szeemann para su exposición Der Hang zum Gesamtkunstwerk, un proyecto que itineró por Zurich, Düseldorf, Viena y Berlín entre 1983 y 1984. Esta reconstrucción contó con la ayuda y memoria del hijo del artista para realizarse, junto a las fotografías que se tomaron de la versión alemana en 1933 (VV.AA.: 1989, p. 172). 
9. Traducción de la autora.

10. Traducción de la autora.

11. Los casos más interesantes generados a finales de los años 20 son, amén de la sala constructivista del Landesmuseum de Hannover, el MoMA de Nueva York y el menos célebre Muzeum Sztuki en Lodz (Polonia).

12. Hemos sido testigos, como espectadores, de dos re-exposiciones de estas copias de 1988 . La que el MNCARS realizó en el proyecto antológico sobre Hannah Höch comisariada por Juan Vicente Aliaga en el año 2004, y la que, organizada por el K20 de Düsseldorf bajo el título La otra cara de la Luna (Die Andere Seite des Mondes) reunía a finales de 2011 la obra de varias artistas mujeres de las Vanguardias Históricas. En el primer caso, una campana protegía las muñecas, sentadas sobre una elevada peana de madera vista y tapando un foto montaje enmarcado, imitando en su presentación la que se muestra en las fotografías originales que se conservan de la Dada Messe berlinesa. En el segundo, una alta vitrina de tipo armario las guardaba colgadas de hilos como si fueran marionetas.

13. Plight tuvo su origen en un dibujo previo realizado por Josef Beuys en 1958, aunque no presentó una formalización hasta 1985, de ahí que se feche 1958-1985. El propio autor subraya con este dato que la obra es un concepto, por encima de todo. La instalación fue provocada por una situación concreta, ya que la galería Anthony d'Offay en Londres, donde el artista alemán estaba proyectando una exposición, sufría esos días de infernales ruidos debidos a la demolición de un edificio vecino. Beuys, que ya estaba enfermo (murió al año siguiente) y que no lograba hacerse oír tras el ruido del martillo neumático, decidió proteger la galería con los cilindros de fieltro (Hohlfeldt: 1999).

14. Traducción de la autora. 